\title{
LIFE PREDICTION FOR RANDOM LOAD FATIGUE BASED ON THE GROWTH BEHAVIOR OF MICROCRACKS
}

H. H. Heitmann, H. Vehoff and P. Neumann

Max-Planck-Institut fuer Eisenforschung, 4000 Duesseldorf, Federal Republic of Germany

ABSTRACT

A crack initiation life prediction scheme is proposed by integrating a growth law for micro-cracks. The growth of small cracks is described by the effective cyclic J-integral, $Z$. From this procedure a new damage parameter, $\mathrm{z}_{\mathrm{d}}$, is defined which contains the plastic strain range, the stress range, and the mean stress. The latter two improve the prediction in the high cycle regime. In order to carry out this type of life prediction a couple of specimens are required to obtain cyclic stress strain curves from incremental step tests and about 10 specimens to obtain an SN-curve in terms of the $z_{d}$ parameter. No crack growth data are required because of the intimate connection between life and crack growth law. Experimental results on mean stress effects and on random loading are compared with the predictions using $z_{d}$ as well as the Smith-parameter.

KEYWORDS Fatigue, crack initiation, small cracks, crack propagation, cyclic
J-integral, random loading, Masing behavior, damage accumulation, life prediction,

INTRODUCTION

Many proposals have been made for predicting the fatigue life under variable amplitude loading on the basis of constant amplitude loading data. Most of these approaches are based on phenomenologically defined damage parameters, which are supposed to determine the increment of damage done to the material within each cycle (Miner's law). In such approaches the physical nature of the fatigue damage as well as of the damage parameters remain unspecified. In the present paper it was tried to avoid both drawbacks by defining the fatigue damage as the maximum crack length present. Life predictions can then be made by integrating the appropriate general crack growth law. Studies of this kind were done previously (Kaisand and Mowbray, 1979, Tanaka, Hoshide, and Maekawa 1982) but were not applied to random loading. On the other hand this study is limited to life predictions on smooth specimens only. 
EXPERIMENTAL

Two low alloy structural steels, StE 47, StE 70 , and one quench and tempered steel 38NiCrMoV 73 (AISI 4340) were used. The latter one was heat treated to two different yield strengths of 740 and $1100 \mathrm{MPa}$. Smooth tensile specimens with a diameter, $\mathrm{D}$, of 6 or $8 \mathrm{~mm}$ and a gauge length of 2D were machined with the specimen axis perpendicular to the rolling direction. gauge length was polished mechanically.

The specimens were fatigued in a computer controlled hydraulic closed-loop that better than $10^{-5}$. straln an accuracy

The random load tests were performed by repeating a quasi-random total strain were distributed 1000 reversal points. The strains at the reversal points $\rho=1 / \varepsilon^{*} \exp \left(-\varepsilon / \varepsilon^{*}\right)$ according to a clipped distribution function and large plast

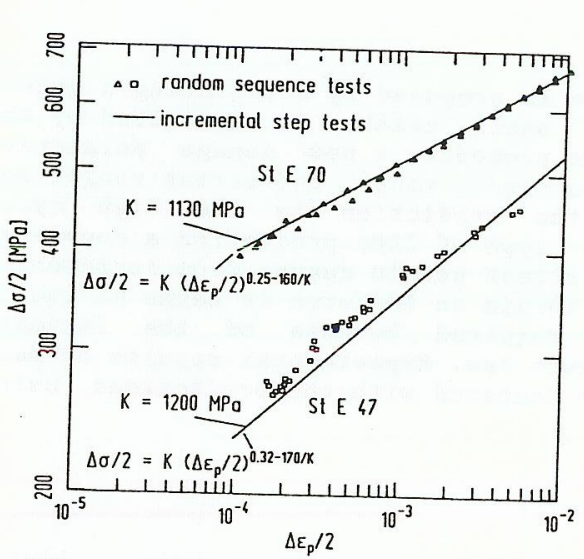

Fig. 1. Stress range versus strain range of closed hystereses loops incremental step tests.
St E 47

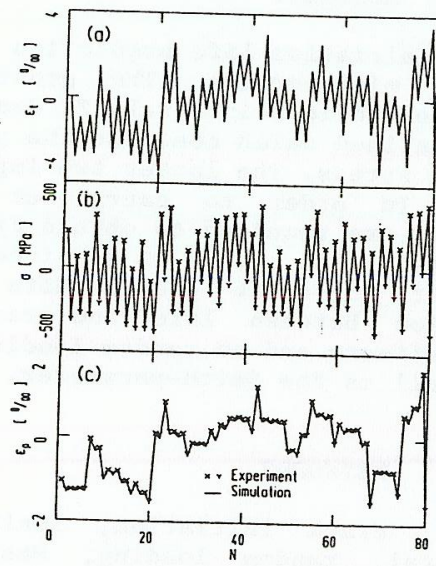

ig. 2. Predicted and measured plastic strain ranges during random total strain tests.
CYCLIC STRESS STRAIN BEHAVIOR.

Usually the loading history is given in terms of the reversal values of either the stress or the total strain. On the other hand the plastic strain is litude is a very important quantity for estimating the fatigue damage is obvious from the fairly general validity of Coffin-Manson's law ( 1953, Coffin 1954). Therefore, as a first step a prediction of the (Manso strains from the given stress or total strain history must he perfor plastic was done by using the well-known Masing-model of use performed. This behavior, which is characterized by the existon strain the so-called cyclic stress strain curve, which (between reversal points) of stress and

Increasing monotonically (Masing 1926). For general loading histories the cyclic memory effect is assumed which enforces rain-flow counting of the closed hystereses loops (Martin, Topper, and Sinclair 1970).

The three steels used show a Masing-type behavior to a reasonable degree of accuracy. The main problem is cyclic softening which is not included in the Masing-model. Because of deviations from the ideal Masing behavior cyclic stress strain curves depend on the exact way how they are measured. Those which were obtained by incremental step tests (Landgraf, Morrow, and Endo 1969) were found to be most similar to the relation between stress ranges and plastic strain ranges during random loading. Both are compared in fig. 1. If strong cyclic softening is observed, however, incremental step test and random loading must have comparable maximum loads to obtain a reasonable agreement. Obviously the cyclic stress strain curve can be approximated by a power-1aw

$$
\Delta \varepsilon_{p}=c_{1} \Delta \sigma^{n}
$$

The two material parameters $C_{1}$ and $n$ can be measured by an incremental step test with a single specimen. Figure 2 shows an example of the prediction of the peak values of the plastic strain calculated from the peak values of the total strain. It is obvious that there is a good correlation with the measured values. In the following we shall use therefore only cyclic stress strain curves obtained from incremental step tests.

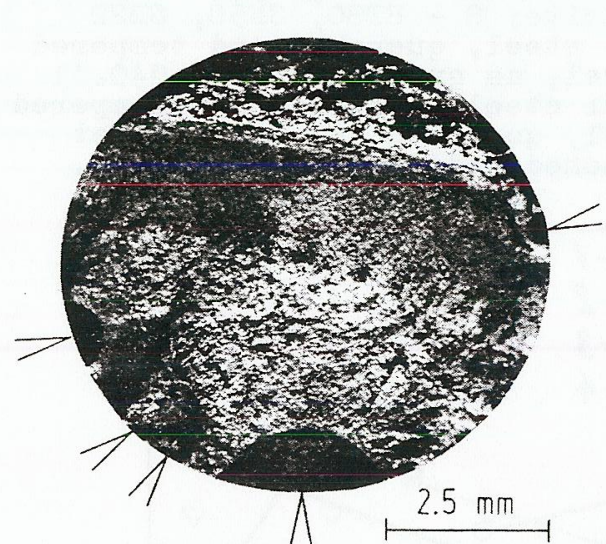

Fig. 3. Semi-circular surface cracks marked by heat tinting on a fracture surface.

THE GROWTH OF MICRO CRACKS.

During random loading microcracks are obtained as soon as the first high peak load is applied. Thus more than $90 \%$ of the technical crack initiation life $(a \leqslant 1 \mathrm{~mm})$ is spent by growth of these microcracks. They originate at surface inclusions ( $\mathrm{rrS}$, or more complex inclusions) which are about $30 \mu \mathrm{m}$ in diamater. Initially the cracks are of comparable size. Depending on the load level, there are many crack nuclei (fig. 3 ), which grow independently as long as their lengths are smaller than their mutual distances. This was found by observing the growth of many individual cracks with the help of an optical 
microscope. The cracks have a well defined almost semi-circular shape with of the local (Raju and Newman 1979).

As shown in fig. 4 the growth behavior of such short described in terms of $\Delta \mathrm{K}_{\text {eff }}$-values. The usual fhort cracks cannot b compared to long cracks is observed, because linear fracture applicable to this case of small cracks embedded in mechanics is no specich a case in uni-directional loading the J-integ plastifled to fo cycling loading of Masing-type materials (Lamba 1975, Wuethrich transferred follow the arguments of Dowling 1977, who estimated the J-values 1982) w surface cracks. The cyclic J-integral, which we she J-values for shor Wuethrich, 1982, in order to distinguish it from the J-Integral, is composed of a plastic, $W_{p}$ and an elastic, $W_{e}$, part accorda to

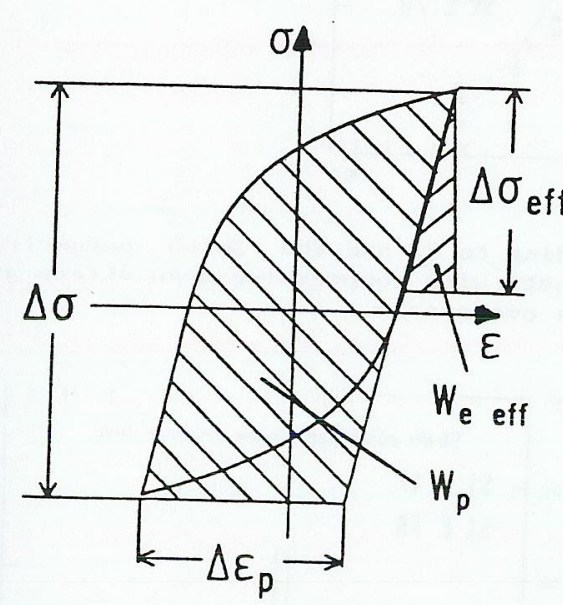

Fig. 5. Definition of the elastic effective cyclic J-integral, $Z_{\text {eff }}$.

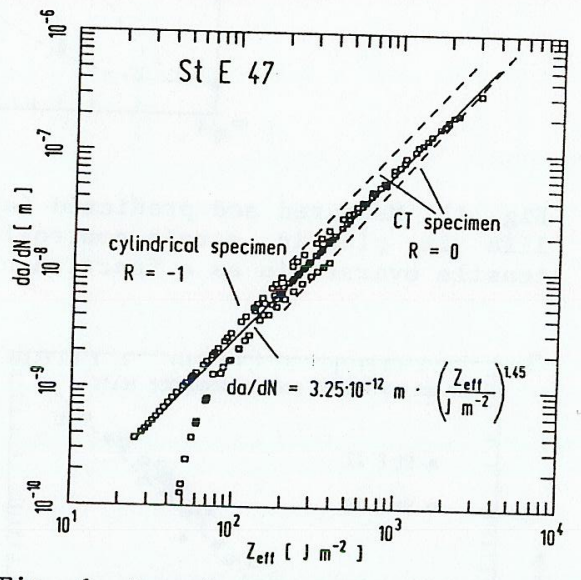

Fig. 6. Growth rate of small cracks as a function of the effective cyclic
J-integral $z$.
With more accurate solutions than those available to Dowling we obtained the following values for the numerical coefficients $f$ ing we obtained the $\mathrm{f}_{2}=2.5$. The definition of $\mathrm{W}_{\mathrm{p}}$ and $\mathrm{W}_{\mathrm{e}}$ in terms of $\mathrm{f}_{1}$ and $\mathrm{f}_{2}: \mathrm{f}_{1}=2.9$ coincides in Masing type materials with the doubled cyclic se loop, which given in fig. 5. Thus the loop shape is given by (1) and we strain curve, are

$$
W_{e} \text { eff }=\frac{\Delta \sigma_{\text {eff }}^{2}}{2 E} \quad W_{p}=\frac{n}{n+1} \Delta \sigma \Delta \varepsilon_{p}=\frac{C_{1} n}{n+1} \Delta \sigma^{n+1}
$$

Crack closure is assumed for the elastic part, $W_{e}$, only and not for the plastic part, $W_{p}$. The effective stress range due to closure, or the
obtained according to

$$
\Delta \sigma_{\text {eff }}=\Delta \sigma 3.72(3-R)^{-1.74} \quad R=\frac{\sigma_{\min }}{\sigma_{\max }}
$$

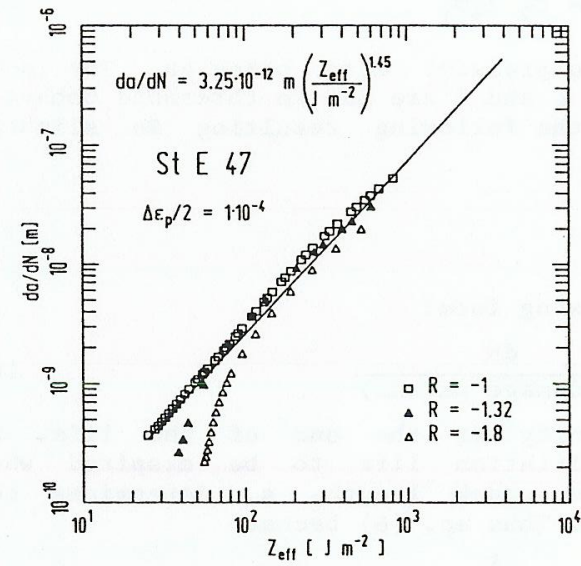

Fig. 7. Crack growth rate of small cracks as a function of the effectiv cyclic J-integral $z_{\text {eff }}$ for three compressive mean stresses.

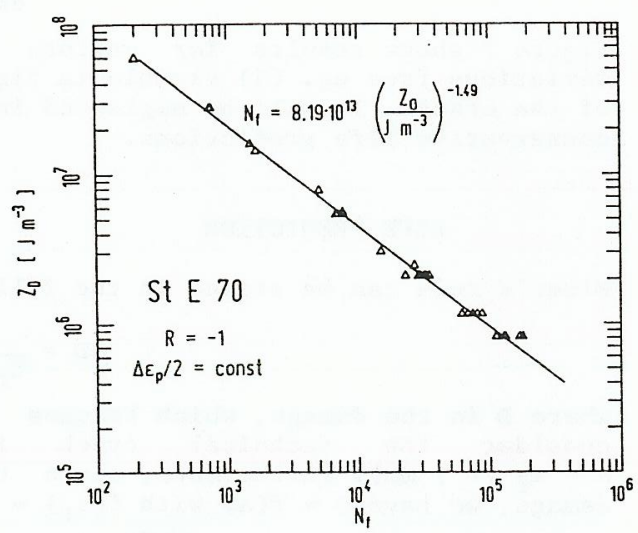

Fig. 8. The technical crack initiation Fig. 8. The technical crack initiation damage parameter $z_{d}$ obtained from constant plastic strain range tests.

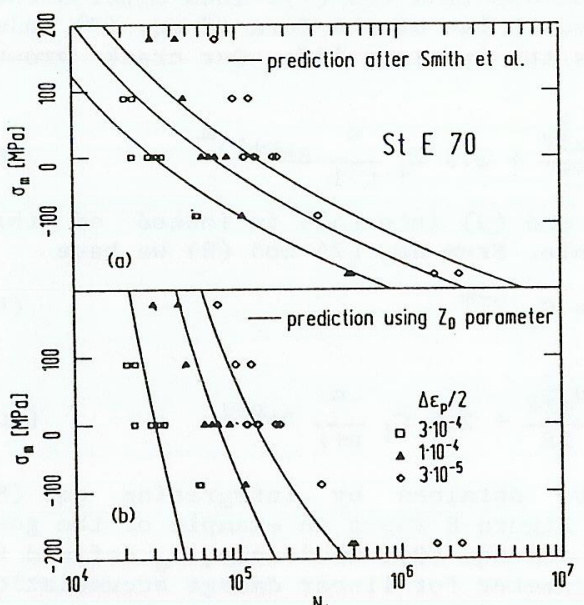

Fig. 9. Cycles to failure as a function of mean stress in constant plastic strain tests. Predictions acoording to Smith et al, and $z_{d}$.

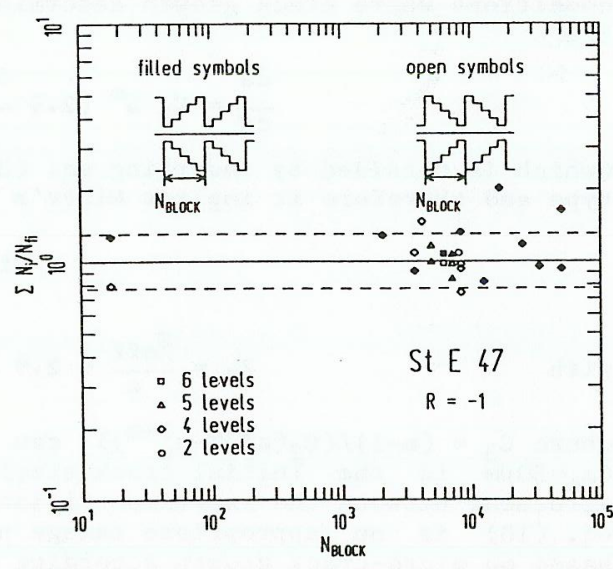

Fig. 10. Calculated damage values at failure for tests with sawtooth loading as a function of the number of cycles within the blocks.

This eq. reproduces Schijve's equation for aluminium (Schijve 1981) for $\mathrm{R}>-1$ and extends it to compressive mean stresses without yielding negative values for $\mathrm{R} \leqslant-1$. Figure 6 shows the crack growth data as a function of
$\mathrm{Z}_{\text {eff }}$. Obviously the data from short as well as long cracks fall on a common 
line in this double logarithmic plot indicating a power-law dependence "I
da/dN on $Z_{\text {eff }}$.

$$
\frac{\mathrm{da}}{\mathrm{dN}}=\mathrm{C}_{2} z_{\mathrm{eff}}^{\mathrm{m}}
$$

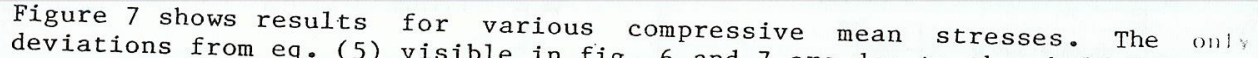
deviations from eq. (5) visible in fig. 6 and 7 are due to threshold behavi..., of the cracks. It will be neglected in the following resulting in slight.,
conservative life predictions.

LIFE PREDICTION

Miner's rule can be stated in the following form:

$$
\mathrm{dD}=\frac{\mathrm{dN}}{\mathrm{N}_{\mathrm{f}}(\text { damage param. })}
$$

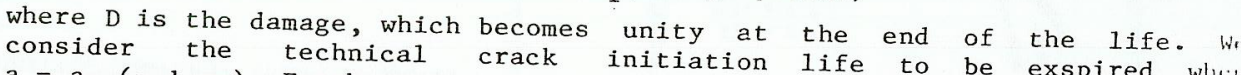
$a=a_{f}(=1 \mathrm{~mm})$. Furthermore, since the crack life to be exspired wh.... $a=a_{f}(=1 \mathrm{~mm})$. Furthermore, since the crack length, a, determines th.
damage, we have $D=f(a)$ with $f\left(a_{f}\right)=1$. Thus eq. (6) becomes

$$
\frac{\mathrm{da}}{\mathrm{dN}}=\frac{1}{\mathrm{df} / \mathrm{da} \mathrm{N}_{\mathrm{f}}(\text { damage param })}
$$

The essential point of the form of eq. (7) is, that the crack lengl dependent part can be separated multiplicatively from the part which depend" is obviously equivalent to a Miner's rul. conditions where crack growth determing law

$$
\frac{\mathrm{da}}{\mathrm{dN}}=c_{2} a^{\mathrm{m}}\left(2.9 \frac{\Delta \sigma_{\text {eff }}^{2}}{2 \mathrm{E}}+2.5 c_{1} \frac{\mathrm{n}}{\mathrm{n}+1} \Delta \sigma^{\mathrm{n}+1}\right)^{m}
$$

(which is obtained by inserting eq. (2) and (3) into (5)) is indeed of thi: type and therefore it implies Miner's rule. From eq. (7) and (8) we have

$$
\mathrm{N}_{\mathrm{f}}=\mathrm{C}_{3} \mathrm{z}_{\mathrm{d}}^{-\mathrm{m}}
$$

with

$$
z_{d}=\frac{z_{\text {eff }}}{a}=2.9 \frac{\Delta \sigma_{\text {eff }}^{2}}{2 E}+2.5 c_{1} \frac{n}{n+1} \Delta \sigma^{n+1}
$$

where $C_{3}=(m-1) /\left(c_{2}\left(a_{f}^{1-m_{-a}} a_{o}^{1-m}\right)\right)$ can be obtained by integrating eq. (8) $\left(a_{0}=30 \mu \mathrm{m}\right.$ is the initial crack size). Figure 8 shows an example of the good agreement between the experimental data and eq. (9). Obvious1y, $z_{d}$ defined by eq. (10) based on micro-crack growth according to eq. (8) $z_{d}$ contains via $W_{\text {and }} \mathrm{w}_{\mathrm{f}}$ necessary stress range as well as the plastic strain range. Both terms are Cofesary in an appropriate damage parameter: The plastic strain term implies parameter in the high cye conditions. The stress term makes $z d$ a valid damage parameter in the high cycle range, where Coffin-Manson's $Z_{d}$ breaks down. Note that no adjustable parameters were used at all and 's law was derived - without any further assumptions - from a crack orowth eq. (10) which in turn was derived from fairly well justified fracture mechanics

Many experiments were performed to test the validity of this prediction method in block loading tests with mean stresses, which are notorlomis difficult to predict (Nowack et al. 1978)). Figure 9 shows the influence of mean stress on the life in tests with constant plastic strain amplitude together with predictions according to the Smith-parameter (Smith et al. 1970 ) and according to $\mathrm{Z}_{\mathrm{d}}$. Obviously $\mathrm{Z}_{\mathrm{d}}$ works better. Figure 10 shows some results from tests with large abrupt changes in the plastic strain amplitude

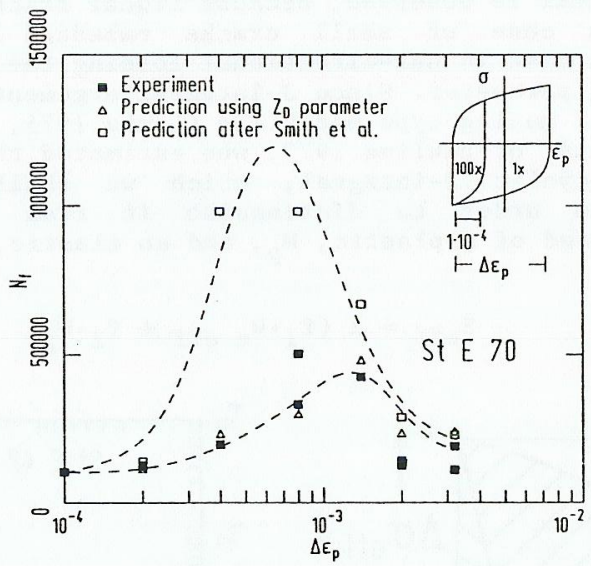

Fig. 11. Measured and predicted (according to $z_{d}$ and the Sinith parameter life in plastic strain controlled tests with compressive men strman and tensile overstrain as a function of the overstrain.

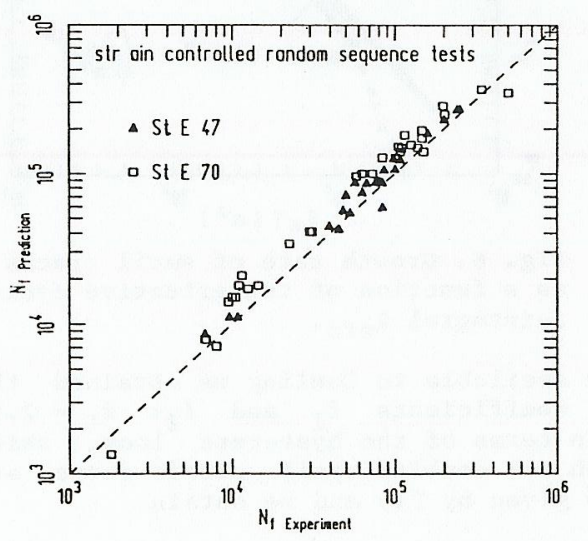

Fig. 12. Comparison of predicted and measured technical crack initiation life for random total strain tests.

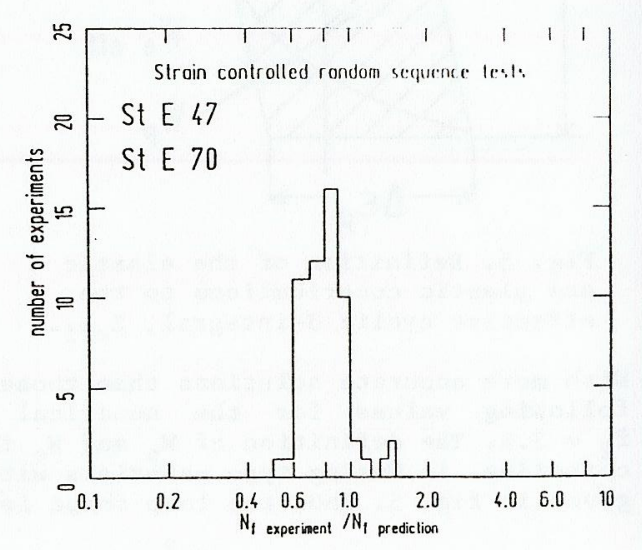

Fig. 13. Distribution function of the ratio between predicted and measured fatigue life in fig. 12 .

in both directions (increasing and decreasing). The plastic strain amplitude varied between $2 \cdot 10^{-5}$ and $10^{-3}$. The calculated damage at failure has tests with compressive 
mean strain and repeated tensile overstrains as a function of the amount of overstrain. The $Z_{d}$ prediction agrees with the data, the Smith-paramater
prediction is less accurate.

Figure 12 shows the predictions for random load experiments with an analysis the scatter in fig. 13. The accuracy of the prediction is of the ander 30\% and is slightly non-conservative.

\section{CONCLUSIONS}

A short guide-line is given how to proceed in order to make predictions of the technical crack initiation life based on $z_{d}$

1. An incremental step test with one specimen yields $c_{1}$ and $n$ in eq. (1). If there is strong cyclic softening, several tests with different maximum loads $\Delta \sigma$ and $\sigma$ performed. This provides all the necessary data to calculate $z_{d}$ from

2. Constant $\mathrm{Z}_{\mathrm{d}}$ fatigue tests (constant $\Delta \varepsilon$ tests are sufficient in mos damage accumulation calculation 1.e. $d \mathrm{D}=\mathrm{dN} /\left(\mathrm{C}_{3} z_{\mathrm{d}}^{-\mathrm{m}}\right)$

seed to be performed because of the

\section{ACKNOWLEDGEMENT}

Financial support from the Bundesministerium fuer Forschung und Technologie, Bonn, under contract no. $03 \mathrm{~S} 1010$ is gratefully acknowledged.

\section{REFERENCES}

Coffin, L.F. (1954). Transaction of the ASME, 76, 931.

Aspects of (1977). In: "Cyclic Stress-Strain and Plastic Deformation Aspects of Fatigue Crack Growth", ASTM STP 637, 97.

Kaisand, L.R. and D.F. Mowbray (1979). J. Test. Eval., 7, 270

Land , H.S. (1975) . Engng. Fract. Mech., 7,693 .

Landgraf, R.W., J.D. Morrow, and T. Endo (1969). J. Mater. Science, 4, 176.

Lankford, J. and F.N. Kusenberger (1973). Met. Trans., 4, 553.

Manson, S.S. (1953). NASA Techn. Note 2933.

Martin, J.F., T.H. Topper, and G.M. Sinclair (1970). Mater. Research, 11, 23. of , G. (1926). In: "Proceedings of the Second International Congress owack H. D. Hans , Zuerich, 332.

berichte der VDI Zeitschriften 18:-H. Trautmann (1978). Fortschritts-

Schijve, J. (1981). Enman (1979). Engng. Fract. Mech., 11, 817

Smith, K.N., P. Watson and T. Topper 14,461

Tanaka, K., T. Hoshide, and 0.

Wuethrich, C. (1982). Int. 